\title{
Integrating Concept-Mapping into Augmented Reality Learning System for Natural Science
}

\author{
Chien-Hsu Chen ${ }^{+}$, Yin-Yu Chou and Chun-Yen Huang \\ Department of Industrial Design, National Cheng Kung University, No.1, University Road, Tainan City 701, \\ Taiwan ROC
}

\begin{abstract}
As the rapid development of technology and the popularity of computer equipments, Computer aided learning makes learning effective and interesting through the use of interactive multimedia technology. In the past decade, augmented reality has become an popular technology and has widely utilized in many researches. Many researchers have integrated AR technology into multimedia instructional practices, increasing instructional flexibility and interaction. However, most of augmented reality learning system didn't have the learning scaffold or method to support learning activity, and often been designed from the perspective of developers, without considering the instructors' instructional progress and methods. Therefore, this study proposed an concept-mapping augmented reality learning system whose architecture and implementation are detailed. To evaluate the effectiveness of the proposed system, a questionnaire was given to 36 eleven years old students after their hands-on experience with the system. Results of the questionnaire showed that the proposed AR learning system support students a motivating, pleasant, and satisfying learning experience. Limitation, conclusion and future studies are given.
\end{abstract}

Keywords: augmented reality, mobile learning, tangible interaction.

\section{Introduction}

Computer hardware and software have developed rapidly and have gradually become instructional aids used in elementary schools. Many researchers have integrated computer technology into multimedia instructional practices, increasing instructional flexibility and interaction. In previous years, augmented reality (AR) has attracted attention as an interactive technology that enabled users interact directly with virtual objects through the device in the real world. Many applications have been proposed in various field, such as the treatment of specific phobias [1], education [2], industry design, [3], and gaming [4]. Numerous studies have stated positive opinions and supported the application of AR in various domains because of its positive effects [5][6]. AR technology has been widely adopted in interactive digital learning for presenting instructional content, effectively delivering this content to learners by integrating realistic environments and virtual objects [7]. Billinghurst, Kato, and Poupyrev [8] applied AR technology to design a children's reading material interface called the Magic Book; the developers produced animations using book content and superimposed this information on the book using AR.

Even though AR applications have been proven that AR instructional activities must meet instructional requirements and provide flexibility and innovation [9]. Several problems have been revealed when applying $\mathrm{AR}$ to learning activities. One major problem is that AR learning systems often been designed from the perspective of developers, without considering the instructors' instructional progress and methods. This problem would lead to lacking definitive goals or scaffolds when conducting instructional activities, substantially reducing students' learning participation and motivation [10].

+ Corresponding author. Tel.: + 88662757575

E-mail address: chenhsu@mail.ncku.edu.tw. 
Numerous learning scaffolds use mind tools such as concept mapping, databases, computer simulation programs, or expert systems. Among these mind tools, the concept mapping has been considered as an effective tool for helping learners and instructors represent knowledge structures [11][12]. In this study, an instructional material design scaffold based on concept mapping was proposed. Concept mapping was integrated with AR technology to implement a concept-mapping augmented reality system (C-MARS) for learning. The paper is organized as follows. Section II we talk about the related work. Section III the CMARS has been proposed and the detailed implementation of C-MARS. Section IV discusses the challenges of C-MARS. At last, conclusions in Section V.

\section{Related Work}

\subsection{Augmented Reality}

Azuma [13] specified the three critical conditions of AR: (a) integrating realistic and virtual objects in identical interfacial spaces; (b) providing real-time interactive platforms; and (c) presenting virtual objects in three-dimensional (3D) spaces with which users can interact. AR is a technology that provides users with an environment that is synthesized using combinations of real-world and virtual objects. The images presented using this technology are composed of combinations of real-world and virtual objects, which comprise images, sounds, and objects that stimulate the senses of taste, scent, and touch or vibration [14]. Specifically, users can sense and interact with virtual objects existing in realistic environments while remaining in the real world. The advantage of AR is the near-real-world operation that integrates virtual $3 \mathrm{D}$ and realistic objects or backgrounds using video-recording technology, enabling users to interact with realistic objects by simultaneously operating virtual 3D objects [13],[15]-[17]. In particular, AR simulations approximate and present real-world human-object interactions, thus enabling users to participate through highly natural and direct interaction [18] [19].

\section{2. $\mathbf{A R}$ in Education}

The highly interactive and tangible user interface of AR technology provides a novel interactive learning method, which has gradually become widely used in computer-aided instructional activities [20]. AR can also enable instructors to design instruction materials that present virtual content and objects in realistic environments. Di Serio, Ibáñez, and Kloos [21] applied AR in visual arts curricula indicated that AR positively influences learning motivation and reduces learners' learning disabilities.

Chen and $\mathrm{Su}$ [22] proposed a sketch environment for young children using computer vision and augmented reality. In this study, AR sketch system presents a friendly way for children to learn and appreciate paintings. And this study also found that the sketch system encourages young children to participate and brings the natural painting experience to an educational environment. Chiang, Yang, and Hwang [23] demonstrated an location-based augmented reality learning in inquiry learning activity. They found that the AR-based inquiry learning activities assist and support the students for sharing the knowledge and provide guidance for helping teachers develop effective learning strategies. Kerawalla [18] applied AR instructional materials in elementary science education by using marker-based AR systems for presenting how the earth's orbiting around its own axis creates day and night. However, this study indicated that the lack of appropriate instructional content in AR-learning systems inhibited the improvement of student learning outcomes. Environments, times, and limitations must be considered when developing AR-learning systems, which must also adopt user-centered designs [18].

\section{Concept-Mapping Augmented Reality System (C-MARS)}

\subsection{System Architecture}

In this study, the C-MARS was proposed to help students explore and organize what they learned in the learning course. Fig. 1 (a) shows the system structure of the C-MARS system used in this study. There are five units in the system architecture of this study, including the input and display unit, the encoder and decoder unit, the 3D data unit, the C-MARS learning material and the interaction unit. Each unit of the system is responsible for an important part which is specifically described below. 
- Input and display unit: Learners use the mobile device to manipulate the C-MARS. The camera on the mobile device continuous image capturing speed for live video capturing, is used to scan/capture the information on the marked cards, which is then sent to the tracking unit for further processing. And learners can use the pattern card to interact with the learning system. The system would display immediately feedback according to learners' manipulation.

- Encoder and decoder unit: This unit is responsible for the encoding and decoding of the marked cards. In this study, every characters is first printed on a card for encoding. Vuforia was used for recognizing and tracking the target marked cards, which were selected and uploaded to the Vuforia Target Manager, an online tool for creating and managing databases, and then the corresponding identification target was established. The Unity Extension Application Program Interface (API) was used to create an augmented reality environment and to implement the interaction module between target marked cards. Using Vuforia and the Unity API to develop the C-MARS not only supported the compiler app for an Android or iOS system or other portable mobile device, but its markerless tracking also reduced unnecessary interference and helped the developer quickly build a stable and accurate augmented reality environment. If an image of a particular marked card is recognized, the encoder and decoder process starts to search the 3D database to find out the corresponding 3D object of the marked card. The decoded result is then sent to a 3D data generator unit to generate 3D objects or animations. The unity unit also track the latest positions of marked cards and their 3D objects representations.

- 3D data unit: All 3D objects and animations were designed and built using 3ds Max.

- C-MARS learning material: This unit defines the content of the teaching process and contains the step-by-step instruction of how to organize the knowledge content. A concept mapping of learning structure for the course was constructed as the core for this system. The learning material of C-MARS is adopted from the topic "Food Chain" of elementary science as the course content.

- Interaction unit: Through this unit, learners can interact with the generated 3D object. This unit defines the interaction and constraints among the various marked cards. According to different behaviors of the learners, the system would response corresponding feedback. For example, When two marked cards are related, the corresponding animation between the two cards would be show on the specific position on the screen.

\subsection{System Implement}

Elder AR systems used head-mounted display (HMD) for displaying 3D objects, many researchers have pointed out that HMDs are expensive, cumbersome, and burdensome for learners to conduct learning activities. Because of the advances of computer hardware and software, this study uses a mobile device to display 3D objects and animations. The proposed C-MARS uses VuforiaTM and Unity software and its script language $\mathrm{C \#}$ for system implementation, and uses $3 \mathrm{ds}$ Max ${ }^{\circledR}$ to design and build the 3D objects and animations.

Fig. 1 (b) shows how the C-MARS works. Learners touched the 3D model of a specific character on the mobile device to learn the specific character's introduction. The virtual line with a red question mark or a solid arrow between two card represents a connection between them. The virtual line means that the connection has not yet been learned, and the solid black arrow means it has been learned. Students completed their concept mapping according to their preferred order of exploration. Based on this procedure, students progressively established an entire knowledge structure for the concept of the food chain course. When they had completed the final learning stages, the processes of the complete ecosystem were displayed. Thus, the students organized and established the course knowledge through high-level system interaction.

\section{Experiment, Evaluation and Discussion}

\subsection{Experiment}

This study ran a pilot experiment for investigating the effect of C-MARS to help learners. There were 36 students from an elementary school in Taiwan participating in this experiment. First, a teacher instructed the basic introduction of the characters. And then, the teacher instructed the related knowledge of the characters, 
and introduced how to manipulate the C-MARS with the marked cards. Then, students used the mobile device to construct and organize their learning knowledge through the step by step way. Finally, students could complete their learning concept mapping using the proposed system.

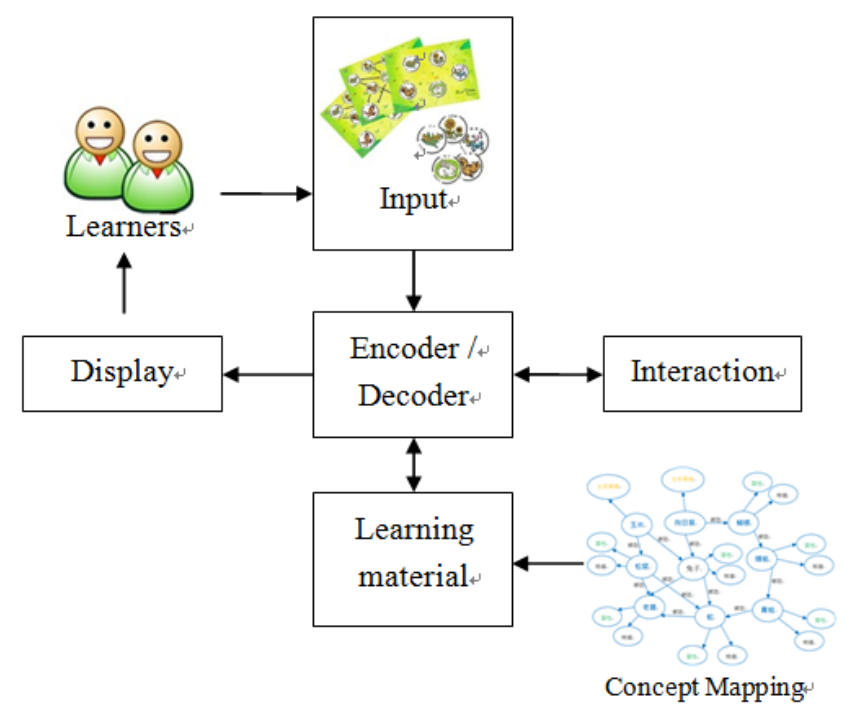

(a)

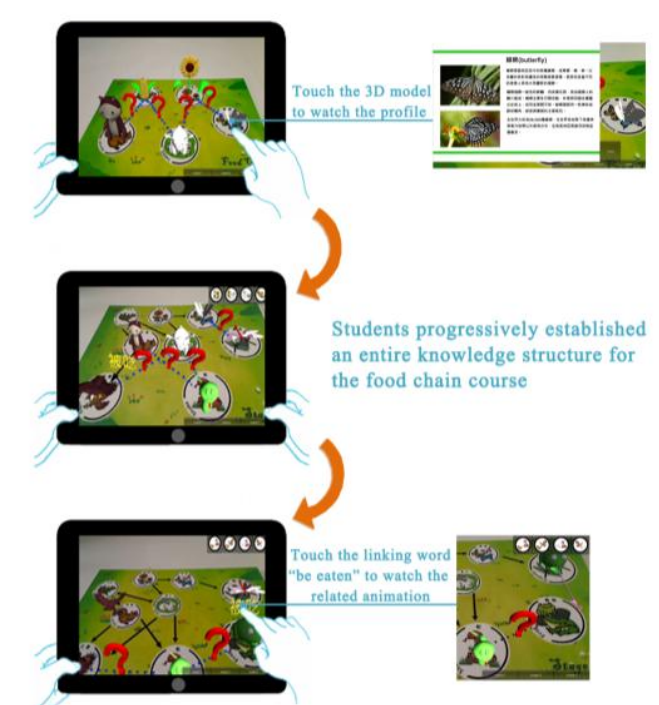

(b)

Fig. 1 : (a)The system architecture of C-MARS. (b): The operating of the system.

\subsection{Evaluation and Discussion}

After the learning experiment, a ARCS learning motivation questionnaire using 5-point Likert scale was given to participants to understand the effect of C-MARS on their learning motivation. Results from the questionnaire showed that the average scores for the subscales of attention, relevance, confidence, and satisfaction were 4.53, 4.42, 4.41, and 4.63 respectively. All scores are above 3.5 indicating that the students has positive learning motivation toward the proposed system. Among the four subscales, the satisfaction subscale was the highest score indicated that students was satisfied with the C-MARS. The second highest score was the attention subscale with the score of 4.53. This might be due to the C-MARS catching the attention of the students, and support they to learn using a clear and simple way.

\section{Conclusion}

AR technology has been widely adopted in interactive digital learning for presenting learning content. Many researches of AR learning used questionnaires to evaluate the effectiveness of AR supported learning, this study adopt pilot study using a questionnaire to understand the students' learning motivation toward the proposed system. Questionnaire results showed that students were positively motivated to learn with the CMARS. From the interview, we found that students were inspired by the C-MARS and wanted to learn more about the related content. There were some unexpected behavior of the students, such as "grabbing" and "slapping" at the 3D characters. This showed that they expected the virtual objects could move just as they do in the physical world. And the students also felt interesting and pleasant about the C-MARS, and they were active and were willing to learn more with C-MARS. Therefore, the C-MARS developed in this study demonstrated that the support of an adequate learning material and scaffold in AR learning when integrating AR technology in course to help student organize their learning content. This study was limited to an investigation within the natural science domain. In the future, we would extend this proposed system to different issue, so more students could utilize AR technology to experience.

\section{References}

[1] C. Botella, J. Breton-Lopez, S. Quero, R. M. Baños, A. Garcia-Palacios, I. Zaragoza. Treating cockroach phobia using a serious game on a mobile phone and augmented reality exposure: A single case study. Computers in Human Behavior. 2011, 27 (1): 217-227. 
[2] E. Hornecker and A. Dünser. Of pages and paddles: Children's expectations and mistaken interactions with physical-digital tools. Interacting with Computers. 2009, 21 (1-2): 95-107.

[3] A. E. Uva, S. Cristiano, M. Fiorentino, and G. Monno. Distributed design review using tangible augmented technical drawings. Computer-Aided Design. 2010, 42 (5): 364-372.

[4] W. Piekarski and B. Thomas. ARQuake: the outdoor augmented reality gaming system. Communications of the ACM. 2002, 45 (1): 36-38.

[5] C. Sandor and G. Klinker. A rapid prototyping software infrastructure for user interfaces in ubiquitous augmented reality. Personal and Ubiquitous Computing. 2005, 9 (3): 169-185.

[6] S. Nicolau, L. Soler, D. Mutter, and J. Marescaux. Augmented reality in laparoscopic surgical oncology. Surgical oncology. 2011, 20 (3): 189-201.

[7] S. H. Lee, J. Choi, and J. I. Park. Interactive e-learning system using pattern recognition and augmented reality. Consumer Electronics, IEEE Transactions on. 2009, 55 (2): 883-890.

[8] M. Billinghurst, H. Kato, and I. Poupyrev. The MagicBook: a transitional AR interface. Computers \& Graphics. 2001, 25 (5): 745-753.

[9] H. Y. Chang, H. K. Wu, and Y. S. Hsu. Integrating a mobile augmented reality activity to contextualize student learning of a socioscientific issue. British Journal of Educational Technology. 2013, 44 (3): E95-E99.

[10] H. K. Wu, S. W. Y. Lee, H. Y. Chang, and J. C. Liang. Current status, opportunities and challenges of augmented reality in education. Computers \& Education. 2013, 62: 41-49.

[11] H. S. Huang, C. C. Chiou, H. K. Chiang, S.-H. Lai, C.-Y. Huang, and Y.-Y. Chou. Effects of multidimensional concept maps on fourth graders' learning in web-based computer course. Computers \& Education. 2012, 58 (3): 863-873.

[12] G. J. Hwang, C. H. Wu, and F. R. Kuo. Effects of Touch Technology-based Concept Mapping on Students' Learning Attitudes and Perceptions. Educational Technology \& Society. 2013, 16 (3): 274-285.

[13] R. T. Azuma. A survey of augmented reality. Presence: Teleoperators and virtual environments. 1997, 6 (4): $355-$ 385.

[14] R. Azuma, Y. Baillot, R. Behringer, S. Feiner, S. Julier, and B. MacIntyre. Recent advances in augmented reality. Computer Graphics and Applications, IEEE. 2001, 21 (6): 34-47.

[15] S. K. Feiner. Augmented Reality: A new Way of Seeing. Scientific American. 2002, 48-55.

[16] B. E. Shelton and N. R. Hedley. Using augmented reality for teaching earth-sun relationships to undergraduate geography students. Proc. of Augmented Reality Toolkit, The First IEEE International Workshop. 2002.

[17] C. H. Chen, I. J. Lee, and L. Y. Lin. Augmented reality-based self-facial modeling to promote the emotional expression and social skills of adolescents with autism spectrum disorders. Research in developmental disabilities. 2015, 36: 396-403.

[18] L. Kerawalla, R. Luckin, S. Seljeflot, and A. Woolard. "Making it real": exploring the potential of augmented reality for teaching primary school science. Virtual Reality, 2006, 10 (3-4): 163-174.

[19] C. H. Chen, I. J. Lee, and L. Y. Lin. Augmented reality-based video-modeling storybook of nonverbal facial cues for children with autism spectrum disorder to improve their perceptions and judgments of facial expressions and emotions. Computers in Human Behavior. 2016, 55: 477-485.

[20] M. Dunleavy, C. Dede, and R. Mitchell. Affordances and limitations of immersive participatory augmented reality simulations for teaching and learning. Journal of Science Education and Technology. 2009, 18 (1): 7-22.

[21] Á. Di Serio, M. B. Ibáñez, and C. D. Kloos. Impact of an augmented reality system on students' motivation for a visual art course. Computers \& Education. 2013, 68: 586-596.

[22] C. H. Chen and C. C. C. Su. Developing an augmented painting interface for enhancing children painting experience. International Journal of Digital Content Technology and its Applications. 2011, 5 (1): 319-327.

[23] T. H. Chiang, S. J. Yang, and G. J. Hwang. An Augmented Reality-based Mobile Learning System to Improve Students' Learning Achievements and Motivations in Natural Science Inquiry Activities. Educational Technology \& Society. 2014, 17 (4): 352-365. 